\title{
Chapter 11
}

\section{Inter-domain Integrity Management for Programmable Network Interfaces}

\author{
Ognjen Prnjat, Lionel Sacks \\ Departement of Electronic and Electrical Engineering, \\ University College London, Torrington Place, London WCIE 7JE, U.K. \\ Phone: +442076793198 Fax: +442073874350 \\ Email: \{oprnjat|lsacks\}@ee.ucl.ac.uk
}

Key words: Programmable networks, Parlay API, multimedia services, integrity methodology, integrity policies.

\begin{abstract}
Rapid development of the programmable network concepts anticipates a new telecommunications environment where the third party service providers will develop sophisticated user-customised multimedia applications and deploy them through the direct access to the network operators' control plane and the network itself. This is a radical change of direction, since currently operators are developing and testing the new services themselves, thus being confident of the correct and proper functioning and performance of the services, support systems and the underlying networks. This future scenario raises the issue of integrity between the network operator and the third party (service provider) domain. Here we discuss the current developments in the area of programmable networks, focusing on the open APIs such as Parlay, and present a top-down methodology for the integrity analysis and the development of integrity policies. Integrity policies give rise to the definition of integrity recommendations to be followed by network operators and service providers, or the definition of testing regimes between the two, and are ultimately enforced by the integrity-preserving mechanisms to be implemented by both parties. Moreover, the reference architecture depicting the approach for deploying the integrity mechanisms and support functionality is given.
\end{abstract}




\section{INTRODUCTION}

Convergence between telecoms and computing is most apparent in the field of programmable networks. Developments such as open network APIs, active networks and mobile agents pave the way for the future environment where the IT world, rich in applications, will cohabit with the telecoms world, rich in networks. By exploiting these technologies, third party application developers and end-users will have the access to the network control and infrastructure traditionally operated and run by the dominant Telcos. This scenario of open network provision is strongly encouraged by the deregulation forces in Europe and the US; and the general market trends towards rapid and efficient development and deployment of user-customised services. The services are becoming diverse, ranging from narrow-band voice telephony through broadband streams to highly dynamic multimedia application support. Demands for services are diversifying, and the requirements of availability and functionality increase as the business and industrial processes become ever more dependent on communications.

Environment is thus forming, in which the $3^{\text {rd }}$ party, value-added service providers, or even end-users, will develop sophisticated applications, exploiting the infrastructure provided by the Telcos, by accessing it at the considerably lower level of control than it was previously possible. Network operators will have little knowledge of the logic of the applications deployed over their control plane, the active packets travelling through their networks, or the pieces of mobile code deployed on their equipment. However, they will require the assurance from the application developers that their applications will not harm the operation of the operators' control systems or the network as a whole. Network operators will require a guarantee that the integrity of their support systems and the network is preserved. Integrity is defined as [Ward95] "ability of the network to retain its specified attributes in terms of performance and functionality". The best-documented integrity breach is the January 1990 brownout [McDo94] of the AT\&T American network. A control mutation originating in the switching system propagated through the signalling network causing degradation of operation and ending in a shut-down. The whole eastern seaboard of the US lost telephone connections for nine hours, with the financial loss of 1 billion dollars. A number of similar integrity breaches followed in Pacific Bell and Bell Atlantic networks in next two years [Hoan93]. Considering these incidents, the established operators rightfully fear to open their networks to less experienced players whose actions might endanger operation of the network.

Integrity issues are crucial in the future telecoms scenarios, where a number of players in the market will have to interwork, on both bearer service and management levels, while having to be confident that this 
interworking will not jeopardise the correct and proper functioning of the networks and systems. This paper discusses the integrity issues likely to be encountered in the future open environment. Programmable network approaches are discussed, with the focus on the open network APIs and the example Parlay API. The scenario of the inter-domain interaction between the network operator and the service provider is then discussed. Finally, we focus on the methodology for management of integrity in the inter-domain, open API-centred environments. Some integrity-preserving policies are discussed, and the reference integrity management architecture is presented.

\section{INTEGRITY ISSUES}

Integrity is a broad term, encompassing a variety of issues concerning system structure, functionality and behaviour. Integrity can be decomposed in a set of attributes [Prnj99a], some of which overlap with certain concepts established in the general computing fields of dependability and safety. Some basic system integrity concepts are outlined below. Note that the system is considered as a set of distributed elements providing a certain service, thus "system" and "service" can be used interchangeably.

Robustness is the ability of the system to handle unexpected events. A system that is robust can cope with all eventualities and continue operations. Reliability is [Reib91]: "the probability of a system performing its purpose adequately for the period of time intended under the operation conditions encountered". Security refers to the ability of the system to detect and avoid intentional external attack. Resilience is the ability of the system to recover from faults. Availability is "the percentage of time during which the system is operational and conforms to its specification" [Vill91]. Performance is the throughput of the system, often traded off against functionality. Scalability is the impact on performance as more entities (processes, devices etc.) are added to the system. Data Coherence: the information copied or distributed through the system needs to remain consistent through time and change of circumstances. Liveness means that something will, eventually, happen: a system might not remain live because it is in a state of either deadlock, when it is expecting an event which cannot occur; or livelock, when it oscillates between a closed set of states that it cannot leave. Feature Interactions refer to when two or more systems, each with well-defined and understood behaviour, result in unforeseen behaviour while operated together [Came93]. Complexity may be an assessment of how long (how many iterations or cycles) an operation takes - known as time complexity. Computational complexity relates to how well a given procedure can be analytically described or determined. Data complexity refers to the 
complexity of data structures and their interdependencies. A high level of complexity, unless it is there to increase robustness, poses more threats to system operation and thus to integrity. A high level of coupling between system components indicates a high level of interdependence: a failure, or an integrity breach, can ripple through the system via the coupling paths. A distinct sub-attribute of integrity is risk: "a measure that combines both the likelihood that the system hazard will cause an accident and the severity of that accident" [Std1228-94]. Here we view risk essentially as $\sim 1$ iintegrity, i.e. the higher the risk, the lower integrity.

The topics described above are all interrelated. For example, a system with poor scalability will loose performance as the number of entities it involves grows. This would reduce availability. Loss of performance and availability can impact on timing of dependent systems, by impacting on data coherence or the liveness constraints. Thus, a framework for analysing the integrity issues and building the desired level of integrity into newly developed systems and applications is of crucial importance. This is particularly important in the future scenarios of programmable networks where the network operators will have no notion of what are the integrity impacts of opening their control and networks to the third parties.

\section{PROGRAMMABLE NETWORKS}

There are numerous approaches to programmable networks. The initial active network concepts are based on data packets which travel through the network carrying programs in their header which can run on network devices such as switches or routers [Tenn96]. These concepts progressed through active bridging [Alex97], where packets carry only the flag indicating the desirability of running a program. The latest initiative in this area is the application-level active networking [Mars99]. Mobile agents are another big arena of research and development, telecoms [Karm97] applications including IN-based service provisioning, network management by delegation [Gold98] and personal communication services. Finally, the network programming interfaces, on which this paper concentrates, provide an open access for service developers to the service components and network control in the operator domain [Laza97]. Using these interfaces, service providers can develop their own applications using the underlying control and network infrastructure provided by the operator.

Main initiatives in the area of programming interfaces are those led by the IEEE Project P1520, which aims at standardising the programming interfaces for networks [Bisw98], and the industry-led Parlay group, which is specifying and developing a new open network application programming 
interface [Parl00]. Parlay specification provides an object-oriented open interface, the Parlay API, to the network generic services in the operator domain. P1520 specifications are somewhat broader, encompassing the CCM interface to the physical element level, the L-interface to the virtual network device level, the $U$ interface to the network generic service level, and the $\mathrm{V}$ interface to the value-addedd services level, while focusing mostly on the $\mathrm{L}$ interface and to some extent the $\mathrm{U}$ interface.

In this paper, we focus on the integrity of interfaces between autonomous domains, that is, the network operator domain and the $3^{\text {rd }}$ party, service provider domain. This interface is referred to as the U interface in the P1520 reference model, and the Parlay API in the Parlay reference model. Common feature of these interfaces is that they essentially specify the interconnection point between the two autonomous domains: network operator and service provider domains. These interfaces can be compared to the ConS and Xuser interfaces in TINA [TINA-BM] and TMN [M.3010] architectures.

\subsection{Parlay inter-domain scenario}

Parlay API is a typical example of a network programming interface. Phase 1 Parlay specification defines an interface for call control (PSTN) and messaging (email, voice), while phase 2, released in January 2000, provides for wireless and IP services. Parlay API is object oriented, extensible (in terms of functionality, management and billing), and network technology independent, providing the user with application portability.

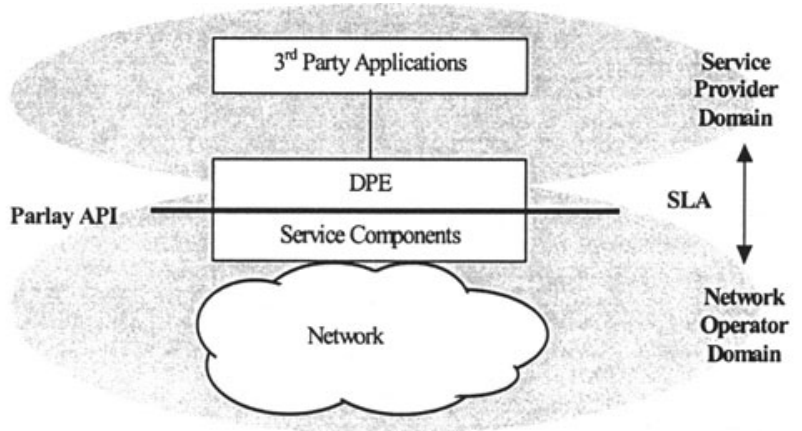

Figure 1. Parlay inter-domain scenario

The context of the use of the Parlay API in the inter-domain interactions is shown on Figure 1. The ellipses represent separate administrative domains. Network operator operates the network resources and the specific service components such as configuration, routing, billing, storage, etc. in its own domain. Operators' service components, and through them the network 
itself, are accessible via the Parlay API. $3^{\text {rd }}$ party service applications access this API, from the service provider domain, through a Distributed Prosessing Environment (DPE), which can be CORBA, JAVA RMI, DCOM or other.

The problem in this context is the preservation of the integral operation of the operators' domain, which can be at stake due to the degre- and logic of the interaction of the $3^{\text {rd }}$ party applications with the service components and sensitive network resources. A number of applications will directly interfere with the network operation, and they may do so with variable frequency and load. Moreover, the network operator has no knowledge of the applications logic and thus can not effectively manage the integrity of its own domain. First step towards the solution to this problem is the establishment of a Service Level Agreement (SLA) between the two players. SLA here has a somewhat broader scope than that of a service conflict resolution tool between the two parties, for what it is mostly used nowadays. SLA is envisaged to embrace the service usage definitions and integrity requirements as well. Considering the issues of service usage, SLA would typically define what operations is the service provider allowed to perform, and the expected resource usage. However, the SLA would also need to define the agreement between the operator and the service provider considering the pure integrity issues, discussed in section 2 . In order to successfully define the integrity issues to be included in the SLA, and develop the ways of ensuring the integrity of the interaction, both players will have to follow an integrity management process.

\subsection{Existing integrity management approaches}

Some solutions to the integrity problem where offered in the area of active networks. Secure Active Network Environment (SANE) [Scot98] is a layered architecture, providing the static checks which ensure that the system starts in the correct state, and the dynamic checks on the per-user or perpacket basis. These checks include authentication, and securing the active node by providing a restricted execution environment for the $3^{\text {rd }}$ party programs, as well as a naming scheme to partition the node resources. Proof Carrying Code (PCC) [Necu97] similarly focuses on the integrity of the active node, by attaching a proof of correctness to each active packet, which is checked at the node before program execution. Finally, a number of active network programming languages, such as Safetynet [Wake] and PLAN [Hick99] aim to ensure integrity at the level of the programming language by tightly controlling the processing time, memory allocation and threading, removing the requirements for run-time checks, and similar.

These solutions seem effective for the protection of the active nodes themselves, but are not fully applicable in the Parlay scenario where the 
programmability is taking place on the interface to the control plane, rather then on the element level. In what follows we present an integrity management methodology and a reference management architecture targeted at the preservation of integrity in the scenario of the open network interfaces.

\section{INTEGRITY MANAGEMENT}

\subsection{Methodology}

The integrity management methodology presented here is based on the methodology introduced in [Prnj99a]. Three phases in this methodology are prediction, testing and maintenance. Prediction is a pre-emptive activity, assessing the features and the overall system integrity status prior to launching. It aims at locating and removing integrity risk areas - hotspots during system development, and building in the integrity preserving mechanisms. Testing is conducted during system development, and as the final phase before the system launch. Testing here refers to the final standalone tests, and tests performed during integration, which are of particular relevance in multi-domain environments. Maintenance is conducted after system deployment and aims at detecting any malfunction or degradation of operation that might pose a risk to system integrity. It involves the measurement of integrity-relevant features of the operational system, diagnostics of cause of degradation, and application of the relevant response.

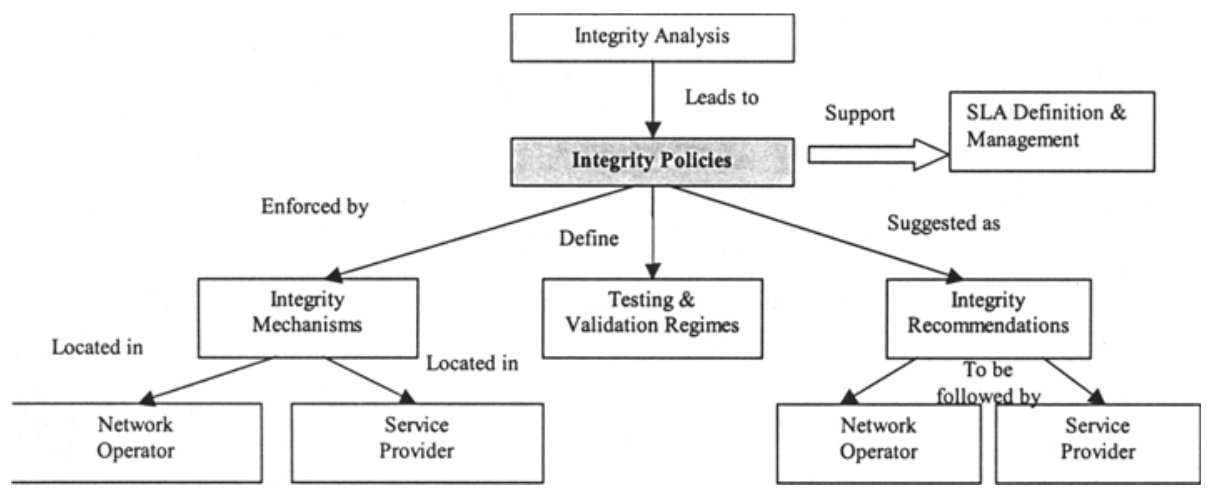

Figure 2. Integrity methodology

Prediction phase is based on the iterative conduction of integrity analysis, and development and implementation of integrity policies throughout the 
system/service development lifecycle. Firstly, analysis of the $3^{\text {rd }}$ party application is conducted, in terms of its own structure and in terms of the interaction with the network operator domain. Integrity analysis takes the issues discussed in section 2 into account, at different stages of development lifecycle. Based on analysis, integrity policies, aimed at preservation of integrity of the interaction between the two domains, are defined. Integrity policies can give rise to definition of the integrity recommendations to be followed by network operators and service providers, or definition of testing regimes between the two (thus leading to the testing phase of the methodology), and are ultimately enforced by the integrity-preserving mechanisms to be implemented by both parties. The mechanisms enforce the policies, and thus belong to the maintenance phase of the methodology. Policies support the SLA definition and management (Figure 2).

Integrity analysis considers a number of integrity issues discussed in section 2. Depending on the stage in system/service development lifecycle, these issues can be analysed either through the use of semi-formal modelling techniques (such as Unified Modelling Language, UML [UML]), or more formal behavioural simulation techniques such as Specification and Description Language, SDL [Z.100]. Detection of the high-risk components early in the development lifecycle can be accomplished using the system metrics [Prnj99b], which pin-point components with high complexity and coupling levels. In its own part, behavioural modelling is of critical importance since the Parlay API specification does not include any behavioural properties, and thus is not robust in terms of operation invocation sequencing and timing that can bring the service components and the network in the undesirable state, causing deadlock or livelock.

Semi-formal and formal modelling for system development is strongly advocated not only as a baseline for integrity analysis. Formal modeling ensures coherent, understandable telecoms system development by providing for unambiguity, completeness and consistency of specifications, and conformance of implementation to specifications; and as such is increasingly used by major players in the telecoms market [Loge99].

\subsection{Integrity policies}

Integrity analysis leads to the definition of integrity policies. These in turn lead to design recommendations to be followed by parties in autonomous domains. They also specify testing and validation regimes, and are ultimately enforced by the integrity preserving mechanisms in the appropriate domains. Some integrity policies can be a responsibility of the DPE used to support the interaction. DPE can sometimes provide for naming, timing, transaction (for preservation of data coherence) and security 
support. Integrity policies can be diverse, depending on the output of the integrity analysis. Some integrity policies are discussed in what follows.

Typical policy that takes a form of a design recommendation is the use of behavioural techniques in specifying not just the logic, i.e. the behaviour of the application, but also the behaviour of the API offered, in the operator domain. Current Parlay API specification is flat: it has the form of UML class diagrams and shows neither the behaviour of the objects implementing the interface, nor the relationships between objects. Some simple UML state diagrams describe the behaviour of few complex objects, and sequence diagrams are specified to guide the developers. This is not enough for specifying and enforcing the correct sequencing of method invocations on the API, and cannot guarantee the preservation of liveness. State diagrams defined for the objects implementing the API interfaces have to be carefully designed and behavioural modelling has to be fully exploited [Kolt00], including reachability analysis, livelock/deadlock detection and robustness.

Data coherence policies support atomicity, consistency, isolation and durability of transactions on data. Example of such integrity policy mechanism is the Transaction Service for network management applications [Ranc98]. This could be the shared responsibility of the DPE.

Policy requiring liveness would employ techniques to detect and resolve any livelock/deadlock states reached by the objects implementing open interfaces. This would typically be the responsibility of the operator. Similarly, integrity mechanisms detecting and resolving feature interactions could be deployed in the operator domain.

Security mechanisms can be used to implement security policies in both domains, dealing with authentication, access control, data integrity, confidentiality and non-repudiation. Current Parlay API Core Specifications provide only the authentication mechanisms through the framework interface. An example [Psou99] security policy would be a reference monitor based on access lists, restricting the access to the service, information, and system resources in the operator domain. This could be further enhanced with limitation techniques for the use of resources in terms of memory, number of subprocesses, CPU time or other. Note that the automatic establishment of the SLA must also be secure: the digital signature (confidentiality and non-repudiation) would be required.

Integrity mechanisms monitoring for performance in terms of throughput and delay can patrol for the compliance with the possible performance-related aspect of the SLA agreement.

Some integrity policies discussed above could be enforced using the authorisation/obligation policy approach as defined in [Slom99]: authorisation policies define what operations can be performed on target objects, and obligation policies define what a manager (in our case the $3^{\text {rd }}$ 
party application) must do as a response to an event trigger. However, some integrity policies have more dynamic properties, in terms of timing, sequencing, performance etc., and are not fully describable using the precondition, parameter and timing constraints defined.

\subsection{Reference integrity architecture and mechanisms}

The reference architecture of Figure 3 depicts the proposed integrity mechanisms which enforce the integrity policies in both service provider and network operator domains; and the additional support integrity functionality. Figure 3 illustrates the same scenario as Figure 1: the two domains are now shown communicating through the API over the DPE. The DPE provides the basic services, like transaction, naming and security (details are dependent on the DPE used). Integrity-supporting elements in the service provider domain include an Integrity Framework (FW) as well as operations and management interfaces (MS) to the operator domain. Operator domain contains the Service Control Point (SCP) for the interface with the control plane, Network Service Emulator (NSE) and Validator (NSV) systems, and the integrity gateway (IG).

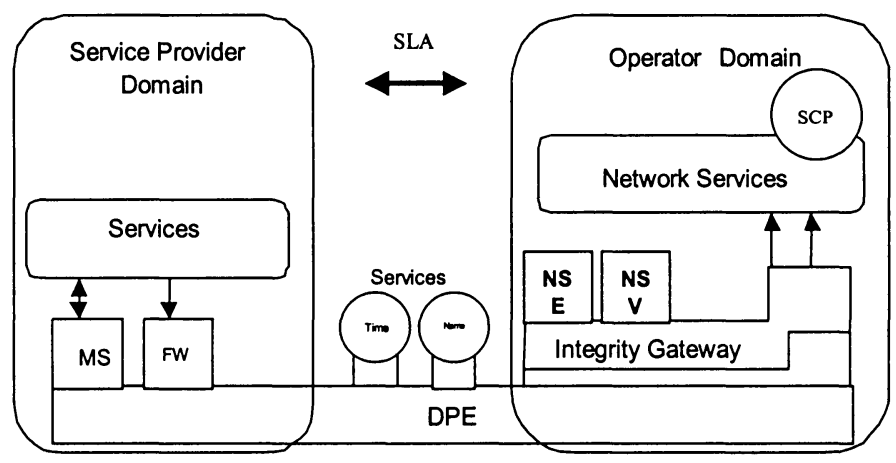

Figure 3. Reference architecture

The integrity gateway (IG) in the operator domain is the key component implementing the integrity mechanisms and thus enforcing integrity policies. Additional policy enforcement is provided by components in the service provider domain - the integrity framework (FW). The objective of the access integrity gateway is to effectively narrow the state and parameter space of requests being made over the open interface to the operator's service platform. The gateway has the characteristics of a service access server by giving access to network services and resources and monitoring for integrity violations. It should provide not only integrity enforcement, but also troubleshooting, violation alarms, event reports and diagnostics. Examples of 
integrity mechanisms implemented in the gateway would be, as discussed in section 4.2, components that monitor system dynamics for livelock or deadlock, data coherence, feature interactions, performance degradations, parameter range violations, resource miss-allocations and such like. Each of these may be dependent on the contractual issues such as: which DPE and DPE services are to be used; which services are being offered, at what successful transaction rate and at which level of robustness. These aspects of the provided service are captured in the SLA.

Some integrity policies give rise to the definition of validation and testing regimes (section 4.1). An overall test environment is a central requirement for the support of many (principally pre-deployment) integrity issues. Focus of this environment is a network service emulator (NSE, Figure 3) capable of taking the place of the actual network control system. This emulator would aid development of service environments and the integrity gateway, without requiring access to a control platform. Further, the emulator would enable services to be tested and validated against integrity requirements prior to deployment. This testing is not envisaged to be exhaustive and delay service roll-out, but tailored to the particular integrity requirements of a particular interaction scenario. In this role the emulator would be developed as a network service validator (NSV, Figure 3 ) and would constitute a clear specific component of the integrity maintenance system. In complement to the validator and the service emulation, a set of service provider-side test suites should be developed to perform pre-production integrity tests on the operator's service provisioning platform. In this way, development of the service emulator not only aids in the development of integrity mechanisms, but establishes a mechanism for testing both the general open network control environments and specific service developments.

\subsection{Inter-domain SLA management}

Different policies for integrity management were discussed in this paper. Depending on the actual scenario of the inter-domain interconnection on the level of the programmable network API, different policies would need to be applied. Policy requirements are specified within the integrity-enhanced SLA established between the two parties in autonomous domains. These requirements would possibly be parametrised, for example as threshold criteria in the form of: "if a certain integrity-related parameter exceeds $\mathrm{x}$, than it is considered as a threat and thus is not acceptable" [Ward95].

Target policies need to be expressed through a formal language. This would ensure the exact and common understanding of the policies by the parties involved in the interconnection, and the automated way of interpreting and enforcing these policies in the integrity gateway. Addition 
of the axioms and inference rules to the formal policy language would yield a formal logic capable of detecting policy conflicts (for some approaches consult [Lupu97]) which may arise due to the inconsistent policy specifications when multiple integrity policies are defined. XML [XML] is being considered as a possible policy language.

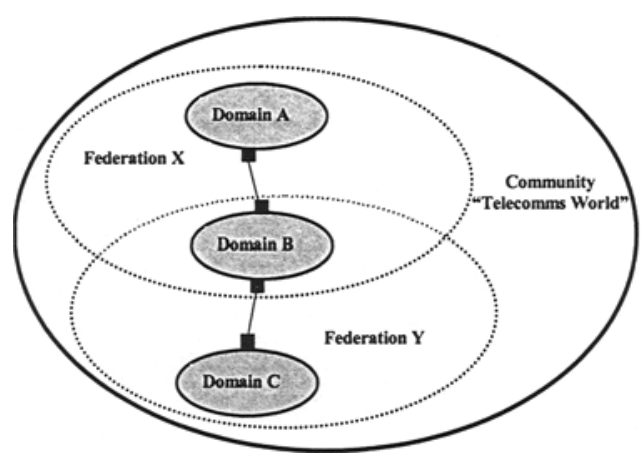

Figure 4. SLAs in federated environments

In the federated environments consisting of multiple autonomous players, the target integrity policies can be established during development and for testing, and the SLAs could be exercised prior to the deployment of $3^{\text {rd }}$ party applications. This approach is more lax in the sense that the integrity policies and SLAs can be established solely by parties willing to indulge in the interconnection. Alternatively, global, regulatory conditions based on quantitative integrity/risk notions can be established by the standardisation bodies and imposed by the regulatory bodies. These two approaches are shown on Figure 4. Domains (autonomous players) are marked as dark ellipses. Federations are marked as grey ellipses, representing groupings of autonomous players with a common objective. Community representing the whole telecoms community is marked by the hollow ellipse. First approach discussed advocates the use of different SLAs (marked by black lines) between domains that form a federation. Federation is based on a certain SLA - a set of integrity rules defining design recommendations, testing regimes, and run-time integrity mechanisms to be deployed. Alternatively, global integrity policies can be established in the telecoms community, governing inter-domain SLA definitions between any two parties.

\section{CONCLUDING REMARKS}

This paper identified some general integrity issues that must be considered in the context of future telecoms scenarios where the networks 
and control systems traditionally managed by the dominant Telcos will be more open to the $3^{\text {rd }}$ party service deployment. An integrity management methodology was presented paving the way for the network operators and third party, value-added application developers and service providers to understand and manage the integrity issues. Focus of the methodology was on the programming network interfaces, such as the Parlay API. The methodology gives rise to definition of: integrity recommendations to be followed by the network operators and application developers; testing and validation regimes to be deployed between the autonomous domains; and implementation of integrity-preserving mechanisms enforcing the integrity policies in both domains. A conceptual reference architecture was presented, encompassing two main integrity enforcing components: integrity gateway in the operator's domain, and the integrity framework in the service provider domain; as well as the service emulator and validator components in the operator domain supporting pre-launch service emulation and validation. The proposed integrity methodology focused strongly on the programming network interfaces, e.g. the Parlay API, and as such is inevitably operatorcentred. Most of the integrity issues in the open API scenarios are solely manageable by the operator. Although existing techniques such as Proof Carrying Code tackle the integrity problem to some extent and put some of the integrity management responsibility on the application developer, they do not resolve issues such as feature interactions, livelock and deadlock detection etc. Authors thus believe that the approach proposed in this paper, although relatively heavy-handed, represents a viable solution in the underresearched field of the programmable interfaces integrity management.

\section{ACKNOWLEDGEMENTS}

Authors would like to thank: Frans Panken, Lucent Technologies; Jeremy Wilson and Martin Yates, BT Labs; Rob Brennan, TelTec DCU; and Ina Schieferdecker, GMD Fokus; for their help in defining some ideas expressed in this paper. This work is funded in part by the EPSRC grant M26091.

\section{REFERENCES}

[Alex97] Alexander et. al., "Active Bridging", Computer Communications Review, 27, 4, pp. 101-111, 1997.

[Bisw98] J. Biswas et. al., "The IEEE P1520 Standards Initiative for Programmable Interfaces", IEEE Communications Magazine, October 1998., pp 64-72.

[Came93] E. J. Cameron and H. Velthuijsen, "Feature Interactions in Telecommunications Systems", IEEE Communications Magazine, August 1993, pp. 18-23. 
[Gold98] G. Goldszmidt and Y. Yemini, "Delegated Agents for Network Management", IEEE Communications Magazine, March 1998, vol. 36, no. 3, pp. 66-70.

[Hick99] M. Hicks et. al., “A Secure PLAN”, Proceedings of the First International Working Conference on Active Networks (IWAN '99), Published by Springer-Verlag.

[Karm97] A. Karmouch, "Mobile Software Agents for Telecommunications", IEEE Communications Magazine Guest Editorial, vol. 36, no. 7, 1997.

[Kolt00] M. Koltsidas, O. Prnjat, L. Sacks, "Development of Parlay-based Applications Using UML and SDL", MMNS' 2000, September 2000.

[Laza97] A. Lazar, "Programming Telecommunication Networks", IEEE Network, October 1997, pp. 2-12.

[Loge99] X. Logean et. al., "On Applying Formal Techniques to the Development of Hybrid Services", IEEE Communications Magazine, Vol. 37, No. 7, July 1999, pp. 132-138.

[Lupu97] E. Lupu and M. Sloman, "Conflict Analysis for Management Policies", Integrated Management IM ' 97 Conference Proceedings.

[M.3010] ITU Rec. M3010, "Principles for a Telecommunications Management Network".

[Mars99] I. W. Marshall, et. al., "Application-level Programmable Network Environment", BT Technology Journal, Vol. 17, No. 2, April 1999.

[McDo94] J. C. McDonald, "Public Network Integrity - Avoiding a Crisis in Trust", IEEE Journal on Selected Areas in Communications, January 1994, vol. 12, no. 1.

[Necu97] G. C. Necula, "Proof-Carrying Code", 24 ${ }^{\text {th }}$ ACM SIGPLAN-SIGACT Symposium Proceedings, 1997.

[Parl00] The Parlay Group, "Version 2.0 Parlay Specification", http://www.parlay.org/

[Prnj99a] O. Prnjat, L. Sacks, "Integrity Methodology for Interoperable Environments", IEEE Communications, May 1999, Vol. 37, No. 5, pp 126-139.

[Prnj99b] O. Prnjat, L. Sacks, "Telecommunications System Design Complexity and Risk Reduction Based on System Metrics", EWDC10, Proceedings published by OCG, 1999.

[Psou99] K. Psounis, “Active Networks: Applications, Security, Safety and Architectures”, IEEE Communications Surveys, First Quarter 1999.

[Ranc98] D. Ranc et al., "Use of Transactions in Network Management Applications", Intelligence in Services and Networks, IS\&N'98 Conference Proceedings.

[Reib91] A. L. Reibman, M. Veeraraghavan, "Reliability Modelling: an Overview for System Designers", IEEE Computer, April 1991.

[Scot98] D. Scott et.al., "A Secure Active Network Environment Architecture", IEEE Network, May/June 1998, vol. 12, no. 3.

[Slom99] M. Sloman, E. Lupu, "Policy Specification for Programmable Networks", Proceedings of IWAN '99 proceedings, Published by Springer-Verlag.

[Tenn96] D. Tennenhouse, D. Wetherall, "Towards an Active Network Architecture", Computer Communications Review, 26, 2, 1996.

[TINA-BM] H. Mulder (Ed.), "Business Model and Reference Points", Version 4.0, www.tinac.com/specifications/specifications.htm

[UML] Rational Software Corporation, Unified Modelling Language, www.rational.com/

[Vill91] A. Villemeur, "Reliability, Availability, Maintainability and Safety Assessment, Vol. 1 "J. Wiley, 1991.

[Wake] I. Wakeman et.al., The Safetynet Project, www.cogs.susx.ac.uk/projects/safetynet/

[Ward95] K. Ward, "Impact of Network Interconnection on Network Integrity", British

Telecommunications Engineering, January 1995, vol. 13, pp. 296-303.

[XML] Extensible Markup Language (XML) W3C Recommendation 10.2.1998.

[Z.100] ITU Rec. Z.100, "Specification and Description Language (SDL)", 1993. 